\title{
Deciphering the immunogenic potential of wheat flour: a reference map of the salt- soluble proteome from the U.S. wheat Butte 86
}

\author{
Susan B. Altenbach ${ }^{1 *}$ (D), Han-Chang Chang ${ }^{1}$ and Annamaria Simon-Buss ${ }^{1,2}$
}

\begin{abstract}
Background: Within the complex wheat flour proteome, the gluten proteins have attracted most of the attention because of their importance in determining the functional properties of wheat flour doughs and their roles in human health conditions such as celiac disease and food allergies. However, certain non-gluten proteins also trigger immunological responses but may be present in flour in low amounts or obscured by the more abundant gluten proteins in two-dimensional gels of total protein preparations.

Methods: Non-gluten proteins were preferentially extracted from the flour with a dilute salt solution and separated by twodimensional gel electrophoresis. Proteins in 173 gel spots were identified by tandem mass spectrometry after cleavage with trypsin or chymotrypsin. Transgenic wheat lines in which specific groups of gluten proteins were suppressed by RNA interference were used to estimate the amount of carry-over of gluten proteins in the salt-soluble protein fraction.

Results: Fifty-seven different types of non-gluten proteins were identified, including 14 types that are known or suspected immunogenic proteins. The predominant proteins in $18 \mathrm{gel}$ spots were gluten proteins. Some of these also contained nongluten proteins. Analysis of the salt-soluble proteins from a transgenic line in which omega-1,2 gliadins were eliminated by RNA interference indicated that certain omega-1,2 gliadins were present in large amounts in the salt-soluble fraction and obscured relatively small amounts of beta-amylase and protein disulfide isomerase. In comparison, analysis of a transgenic line in which alpha gliadins were absent revealed that glyceraldehyde-3 phosphate dehydrogenase was a moderately abundant protein that co-migrated with several alpha gliadins.

Conclusions: In this study, we constructed a proteomic map of the non-gluten protein fraction of wheat flour from the US wheat Butte 86 that complements a proteomic map of the total flour proteins developed previously for the same cultivar. Knowing the identities of low abundance proteins in the flour as well as proteins that are hidden by some of the major gluten proteins on two-dimensional gels is critical for studies aimed at assessing the immunogenic potential of wheat flour and determining which wheat proteins that should be targeted in future gene editing experiments to reduce the immunogenic potential of wheat flour.
\end{abstract}

Keywords: Albumins/globulins, Allergens, Celiac disease, Non-celiac wheat sensitivity, Wheat flour proteome, Mass spectrometry

\footnotetext{
* Correspondence: susan.altenbach@usda.gov

${ }^{1}$ Western Regional Research Center, United States Department of

Agriculture-Agricultural Research Service, Albany, CA, USA

Full list of author information is available at the end of the article
}

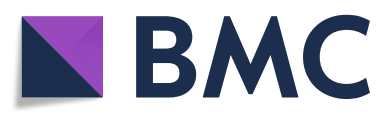

(c) The Author(s). 2020 Open Access This article is licensed under a Creative Commons Attribution 4.0 International License, which permits use, sharing, adaptation, distribution and reproduction in any medium or format, as long as you give appropriate credit to the original author(s) and the source, provide a link to the Creative Commons licence, and indicate if changes were made. The images or other third party material in this article are included in the article's Creative Commons licence, unless indicated otherwise in a credit line to the material. If material is not included in the article's Creative Commons licence and your intended use is not permitted by statutory regulation or exceeds the permitted use, you will need to obtain permission directly from the copyright holder. To view a copy of this licence, visit http://creativecommons.org/licenses/by/4.0/. The Creative Commons Public Domain Dedication waiver (http://creativecommons.org/publicdomain/zero/1.0/) applies to the data made available in this article, unless otherwise stated in a credit line to the data. 


\section{Background}

Wheat flour proteins can be separated on the basis of solubility into the water/salt-insoluble gliadins and glutenins (gluten proteins) and the water/salt-soluble albumins and globulins (non-gluten proteins). The gluten proteins, comprising $\sim 80 \%$ of the total protein, are a collection of $\sim 60-80$ different proteins that serve as the primary storage proteins of the grain while the non-gluten proteins are a diverse collection of different types of proteins that have metabolic, structural or defense functions. Much of the past research has focused on the gluten proteins because of their important role in determining the unique viscoelastic properties that give wheat flour its commercial value. These proteins are also of considerable interest because they are a major trigger of human health conditions, including celiac disease (CD), a chronic inflammatory disease of the small intestine that occurs in genetically susceptible individuals, and food allergies $[1,2]$. The gliadins are primarily monomeric proteins that are divided into four groups termed alpha, gamma, omega and delta gliadins while the glutenins are polymeric proteins that consist of high molecular weight glutenin subunits (HMW-GS) and low molecular weight glutenin subunits (LMW-GS). Epitopes that stimulate T-cells in CD patients have been identified in proteins within most major classes of gluten proteins, although epitopes found in certain alpha gliadins and a subclass of omega gliadins, the omega-1, 2 gliadins, have been found to be immunodominant $[3,4]$. Gluten proteins in all major classes also react with IgE from patients with wheat allergy [5]. In particular, the omega-5 gliadins, another subclass of omega gliadins, have been found to trigger the serious food allergy wheat-dependent exercise-induced anaphylaxis that occurs in sensitized individuals when the ingestion of wheat is followed by physical exercise [6].

Non-gluten proteins also have been found to be immunogenic. In studies that examined cohorts of 28 and 60 patients with confirmed cases of wheat allergy, Battais et al. [5, 7] found that IgE from 67 and $72 \%$ of patients, respectively, reacted with proteins in the albumin/globulin fraction. Further studies using 1-D and 2-D immunoblots of albumin/globulin fractions demonstrated that a wide range of proteins often react with $\mathrm{IgE}$ from single patients and that the reactive proteins vary from individual to individual [8-11]. Non-gluten proteins likely to be allergenic are listed in the Allergome database (http://www.allergome. org/) and include beta amylase (Tri a 17), thioredoxin (Tri a 25), serpin (Tri a 33), triosephosphate isomerase (Tri a 31), peroxidase (Tri a peroxidase), chitinase (Tri a chitinase), peroxiredoxin (Tri a 32), glyceraldehyde 3-phosphate dehydrogenase (GAPDH) (Tri a 34), xylanase inhibitor (Tri a XI), dehydrin (Tri a 35), $9 \mathrm{kDa}$ lipid transfer protein (Tri a 14) and a number of alpha amylase/trypsin inhibitors (AAI) (Tri a 28, Tri a 29, Tri a 30, Tri a 40). In addition, certain non-gluten proteins have been shown to be reactive with IgG and IgA antibodies from CD patients, suggesting a role for serpins, globulins, purinins, farinins and AAI in CD [12]. AAI also have been implicated in non-celiac wheat sensitivity (NCWS), a response to wheat ingestion that is distinct from $\mathrm{CD}$ and food allergies [13], while a globulin from wheat flour has been associated with the development of type 1 diabetes [14].

Because sensitivities to wheat are increasing, there is a need to identify clinically relevant proteins in wheat flour, associate specific flour proteins with particular types of allergies and sensitivities, and determine the frequencies of immunogenic responses to particular proteins. Twodimensional immunoblot analysis is a powerful method for assessing the reactivity of patient sera to specific proteins in the flour $[8,10-12,15-17]$. The approach combines high resolution two-dimensional gel electrophoresis (2-DE), a robust, reproducible method for the both the separation and quantification of hundreds of proteins, with the identification of individual proteins by tandem mass spectrometry (MS/ MS). However, 2-DE analyses of total protein preparations from wheat flour can be problematic because the more abundant gluten proteins overlap with low-abundance nongluten proteins in some areas of the gels [18]. In a comprehensive analysis of total flour proteins, Dupont et al. [19] found MS/MS evidence for non-gluten proteins in $22 \%$ of 2DE spots containing either LMW-GS or gliadins as the predominant proteins. In their study, LMW-GS overlapped with globulins, peroxidase, serpin and xylanase inhibitor; alpha gliadins overlapped with fructose bisphosphate aldolase, alcohol dehydrogenase and formate dehydrogenase; gamma gliadins overlapped with triticin and aspartic proteinase; and omega gliadins overlapped with beta-amylase. For this reason, saltsoluble protein fractions should also be considered for studies of immunogenic proteins. In this paper, we extracted proteins from flour from the US wheat Butte 86 with a dilute salt solution, separated the proteins by 2-DE, and identified proteins in 1732 -DE spots by MS/MS. The resulting proteomic map should make it possible to use 2-D immunoblots to rapidly identify and prioritize non-gluten proteins to be targeted in future gene editing experiments aimed at reducing the immunogenic potential of wheat flour.

\section{Methods}

Proteins were extracted from wheat flour from the US spring wheat cultivar Butte 86 with a buffer containing $100 \mathrm{mM} \mathrm{KCl}$ as described in Hurkman and Tanaka [18], quantified by Lowry, and analyzed by $2-\mathrm{DE}$ as described in detail in Dupont et al. [19]. In short, $50 \mathrm{mg}$ flour was suspended in $200 \mu \mathrm{l}$ of $50 \mathrm{mM}$ Tris- $\mathrm{HCl} \mathrm{pH} \mathrm{7.8,100} \mathrm{mM}$ $\mathrm{KCl}, 5 \mathrm{mM}$ EDTA and incubated for $5 \mathrm{~min}$ with intermittent vortex mixing. Samples were centrifuged at $4{ }^{\circ} \mathrm{C}$ at $14,500 \mathrm{x} \mathrm{g}$ for $15 \mathrm{~min}$. The supernatant was collected and proteins were precipitated by the addition of $4 \mathrm{vol}-$ umes of cold $\left(-20^{\circ} \mathrm{C}\right)$ acetone. The pellet was rinsed 
with cold acetone, air-dried, and stored at $-20^{\circ} \mathrm{C}$. After quantification, proteins were solubilized in urea buffer (9 M urea, 4\% NP-40, 1\% DTT, 2\% ampholytes) for isoelectric focusing using capillary tube gels with a $\mathrm{pI}$ range of 3-10. NuPAGE 4-12\% Bis-Tris protein gels (Life Technologies, Carlsbad, CA, USA) were used for the second dimension separation.

\section{LC-MS analysis}

Protein spots were excised from replicate 2-D gels and placed in 96-well plates where they were reduced, alkylated and digested separately with trypsin (Sequencing Grade Modified Trypsin, Promega Corp., Madison, WI, USA) or chymotrypsin (Sequencing Grade Chymotrypsin, Promega Corp., Madison, WI, USA) using a DigestPro according to the directions of the manufacturer (Intavis, Koeln, Germany).

Plates containing peptides from digested gel spots were placed in the autosampler of a NanoLC 425 (Sciex, Framinham, MA, USA) that was interfaced by a nano-electrospray source to an Orbitrap Elite mass spectrometer (Thermo Scientific, San Jose, CA, USA). Ten $\mu$ l fractions were loaded by the autosampler onto one of three PicoChip reverse phase columns (PicoChip with ReproSil-PUR C18, $10.5 \mathrm{~cm}$, ID $75 \mu \mathrm{m}, 3 \mu \mathrm{m}, 120 \AA$, ReproSil-PUR-C18-AQ, New Objective Inc., Woburn, MA, USA). The positions of the columns were switched in front of the mass spectrometer with the PicoSlide system for Thermo LTQ/Orbi (New Objective Inc., Woburn, MA, USA). With this configuration, Column A is eluting, Column B is loading and Column $C$ is washing, thereby saving time and reducing carryover between samples.

The loaded sample was washed with solvent A to remove salts and the column position was switched in front of the Orbitrap Elite mass spectrometer and eluted with a gradient of acetonitrile into the mass spectrometer. Solvent A was 5\% in acetonitrile and Solvent B was $80 \%$ in acetonitrile, both solvents were $0.05 \%$ in formic acid. Gradient elution was at a flow rate of $250 \mathrm{nl}$ per minute from $100 \%$ Solvent A to $35 \%$ Solvent B in 45 min. Peptides were detected in the Orbitrap set to scan a range from 200 to $2000 \mathrm{~m} / \mathrm{z}$ at a resolution of 60,000 . The 10 most intense peaks were subject to collisioninduced dissociation (CID). The minimal signal threshold was set to 10,000 . Dynamic exclusion with a repeat count of 2 was enabled for duration of $10 \mathrm{~s}$. Normalized collision energy was set to $30 \%$.

\section{Data analysis}

The vendor specific raw files were converted into .mgf files using MSConvert (ProteoWizard, Palo Alto, CA, USA). Spectral data was searched against a database containing all Triticeae sequences from NCBI downloaded on 02/07/2018, full-length Butte 86 sequences from Dupont et al. [19], full length Chinese Spring gluten protein sequences reported by Huo et al. [20, 21], and common MS contaminant sequences contained in the common Repository of Adventitious Proteins (cRAP) database (ftp://ftp. thegpm.org/fasta/cRAP/crap.fasta). Two search engines were used for analysis, Mascot (www.matrixscience.com) and XTandem! (https://www.thegpm.org/TANDEM/).

Results were loaded into Scaffold 4 (Scaffold_4.9.0, Proteome Software, Inc., Portland, OR, USA). The mass accuracy was set to $30 \mathrm{ppm}$ on precursor level and 0.6 Da on MS/MS level. Scaffold summarized the Mascot and X!Tandem peptide hit results from the two different enzyme digestions (trypsin, chymotrypsin) with the MudPit analysis and compress data summarizing option.

Twenty-one spots that contained evidence of gluten proteins in this analysis were excised from replicate gels, digested with thermolysin (Promega Corp., Madison, WI, USA), and analyzed by MS/MS as above. These included spots $26,27,45,54,55,60,61,80,82,83,84,87,91,101$, $102,104,105,106,189,190$, and 191. Data from all three enzyme digestions were then combined in Scaffold. The predominant protein in each spot was deemed to be the protein for which the greatest number of unique peptides were identified and is reported in Table 1. All proteins identified in each spot are reported in Additional file 1. MS data for predominant proteins and proteins that contained at least half the number of unique peptides found in the predominant proteins are shown in Additional file 2. The mass spectrometry data have been deposited to the ProteomeXchange Consortium (http://proteomecentral. proteomexchange.org) via the PRIDE partner repository [23] with the dataset identifier PXD017260 and https:// doi.org/10.6019/PXD017260.

Flour from transgenic lines missing specific gluten proteins was used to further evaluate spots containing both gluten proteins and non-gluten proteins. Transgenic lines were produced previously using RNA interference. Transgenic line SA-30-118a-5 is missing all omega-1,2 gliadins [16] while transgenic line SA-35a$124 \mathrm{j}$ is missing all alpha gliadins [17].

\section{Results}

$\mathrm{KCl}$-soluble proteins from Butte 86 flour were separated by $2-\mathrm{DE}$ and the pattern was compared to that of a total SDS protein extract (Fig. 1). The most abundant proteins in the total protein extract, representing 125 of $2332-\mathrm{DE}$ spots identified previously by MS/MS [19], were the gluten proteins; the HMW-GS and LMW-GS (shown in green), the alpha and gamma gliadins (shown in blue) and the omega gliadins (shown in red) (Fig. 1a). Several groups of non-gluten proteins, including serpins, purinins and alpha-amylase/trypsin inhibitors (AAI), were wellseparated from the major gluten proteins but of considerably lower abundance. In comparison, the most abundant 
Table 1 Predominant proteins identified by MS/MS in 2-DE protein spots from the $\mathrm{KCl}$-soluble fraction of Butte 86 flour. All proteins had identification scores greater than 99\% probability with at least 4 peptides as determined by Scaffold. Positions of spots in 2-DE are shown in Fig. 2. Identities of all proteins found in each spot are reported in Additional file 1

\begin{tabular}{|c|c|c|c|c|c|c|}
\hline Spot Number & Predominant Protein & Accession $\#^{a}$ & Source & \# Unique peptides & \# Spectra & $\%$ Coverage \\
\hline 1 & pyruvate phosphate dikinase & XP_0201883631 & Ae. tauschii & 19 & 50 & 24 \\
\hline 2 & pyruvate phosphate dikinase & XP_0201883631 & Ae. tauschii & 19 & 83 & 21 \\
\hline 3 & pyruvate phosphate dikinase & XP_0201883631 & Ae. tauschii & 20 & 101 & 26 \\
\hline 4 & aconitate hydratase & XP_020176302 & Ae. tauschii & 13 & 31 & 15 \\
\hline 5 & no ID & & & & & \\
\hline 6 & heat shock protein 101 & AAD22629 & T.aestivum & 26 & 67 & 31 \\
\hline 7 & elongation factor 2 & EMS59408 & T. urartu & 20 & 52 & 25 \\
\hline 8 & elongation factor 2 & EMS59408 & T. urartu & 11 & 25 & 13 \\
\hline 9 & embryonic protein DC-8-like & XP_020196294 & Ae. tauschii & 14 & 50 & 24 \\
\hline 10 & embryonic protein DC-8-like & XP_020196294 & Ae. tauschii & 20 & 59 & 29 \\
\hline 11 & embryonic protein DC-8-like & XP_020196294 & Ae. tauschii & 17 & 65 & 31 \\
\hline $12^{b}$ & embryonic protein DC-8-like & XP_020196294 & Ae. tauschii & 30 & 85 & 38 \\
\hline 13 & embryonic protein DC-8-like & XP_020196294 & Ae. tauschii & 42 & 131 & 50 \\
\hline 14 & heat shock protein 90 & ABG57075 & T.aestivum & 19 & 64 & 26 \\
\hline 15 & heat shock protein 70 & XP_020151532 & Ae. tauschii & 43 & 209 & 48 \\
\hline 16 & heat shock protein 70 & EMS51416 & T. urartu & 26 & 85 & 35 \\
\hline 17 & no ID & & & & & \\
\hline 18 & beta-D-glucan exohydrolase & AAM13694 & T.aestivum & 25 & 84 & 34 \\
\hline 19 & beta-D-glucan exohydrolase & AAM13694 & T.aestivum & 24 & 60 & 33 \\
\hline 20 & beta-D-xylosidase & XP_020155854 & Ae. tauschii & 17 & 44 & 23 \\
\hline $21^{c}$ & glucose and ribitol dehydrogenase & pir T06212 & Hordeum vulgare & 7 & 19 & 28 \\
\hline 22 & no ID & & & & & \\
\hline 23 & glucose-6-P isomerase & ABE41790 & T.aestivum & 22 & 67 & 37 \\
\hline 24 & glucose-6-P isomerase & ABE41790 & T.aestivum & 21 & 59 & 38 \\
\hline 25 & globulin 3A & AFM30909 & T.aestivum & 12 & 38 & 24 \\
\hline $26^{b, c}$ & beta-amylase & XP_020197275 & Ae. tauschii & 64 & 397 & 70 \\
\hline $27^{c, d}$ & beta-amylase & XP_020197275 & Ae. tauschii & 46 & 287 & 58 \\
\hline 28 & beta-amylase & EMS68884 & T. urartu & 21 & 83 & 37 \\
\hline 29 & beta-amylase & EMS68884 & T. urartu & 22 & 88 & 40 \\
\hline 30 & ketol-acid reductoisomerase & XP_020174215 & Ae. tauschii & 38 & 111 & 47 \\
\hline 31 & leucine aminopeptidase 2 & EMS53149 & T. urartu & 26 & 61 & 47 \\
\hline 32 & atp1 & ACA62607 & T.aestivum & 20 & 67 & 38 \\
\hline 33 & aldehyde dehydrogenase & AKE36953 & T.aestivum & 6 & 17 & 16 \\
\hline 34 & omega gliadin & AKB95614 & T. urartu & 18 & 34 & 51 \\
\hline 35 & enolase & XP_020163593 & Ae. tauschii & 33 & 135 & 78 \\
\hline 36 & enolase & XP_020163593 & Ae. tauschii & 55 & 164 & 89 \\
\hline 37 & enolase & BAJ85279 & Hordeum vulgare & 45 & 167 & 83 \\
\hline $38^{c}$ & enolase & BAJ85279 & Hordeum vulgare & 19 & 62 & 63 \\
\hline 39 & alanine aminotransferase & XP_020147857 & Ae. tauschii & 38 & 104 & 66 \\
\hline 40 & leghemoglobin reductase & XP_020185250 & Ae. tauschii & 35 & 139 & 69 \\
\hline 41 & cupincin & XP_020147625 & Ae. tauschii & 16 & 42 & 33 \\
\hline 42 & cupincin & XP_020147625 & Ae. tauschii & 30 & 90 & 47 \\
\hline
\end{tabular}


Table 1 Predominant proteins identified by MS/MS in 2-DE protein spots from the KCl-soluble fraction of Butte 86 flour. All proteins had identification scores greater than $99 \%$ probability with at least 4 peptides as determined by Scaffold. Positions of spots in 2-DE are shown in Fig. 2. Identities of all proteins found in each spot are reported in Additional file 1 (Continued)

\begin{tabular}{|c|c|c|c|c|c|c|}
\hline Spot Number & Predominant Protein & Accession $\#^{a}$ & Source & \# Unique peptides & \# Spectra & $\%$ Coverage \\
\hline 43 & cupincin & XP_020147625 & Ae. tauschii & 35 & 105 & 51 \\
\hline 44 & cupincin & XP_020147625 & Ae. tauschii & 33 & 151 & 47 \\
\hline $45^{d}$ & cupincin & XP_020147625 & Ae. tauschii & 35 & 192 & 53 \\
\hline 46 & cupincin & XP_020147625 & Ae. tauschii & 38 & 112 & 53 \\
\hline 47 & globulin-3A & AFM30909 & T.aestivum & 34 & 136 & 45 \\
\hline 48 & globulin-3A & AFM30909 & T.aestivum & 35 & 107 & 38 \\
\hline 49 & globulin-3A & AFM30909 & T.aestivum & 43 & 147 & 46 \\
\hline 50 & globulin-3A & AFM30909 & T.aestivum & 25 & 77 & 28 \\
\hline 51 & translation elongation factor 1 alpha & AAA34306 & T.aestivum & 25 & 109 & 44 \\
\hline 52 & globulin 3 & ACJ65514 & T.aestivum & 5 & 13 & 8 \\
\hline 53 & globulin-3A & AFM30909 & T.aestivum & 25 & 83 & 28 \\
\hline $54^{\mathrm{d}, \mathrm{e}}$ & globulin-3A & AFM30909 & T.aestivum & 23 & 107 & 35 \\
\hline $55^{\mathrm{d}, \mathrm{f}}$ & LMW-GS & ACA63869 & T.aestivum & 27 & 84 & 53 \\
\hline 56 & globulin-3A & AFM30909 & T.aestivum & 7 & 23 & 14 \\
\hline 57 & LMW-GS & ABC84366 & T.aestivum & 28 & 54 & 60 \\
\hline 58 & LMW-GS & ABC84366 & T.aestivum & 9 & 14 & 29 \\
\hline 59 & ERBB-3 binding protein & XP_020191236 & Ae. tauschii & 11 & 53 & 29 \\
\hline $60^{d}$ & gamma gliadin & BU-gamma-5 & T.aestivum & 15 & 38 & 41 \\
\hline $61^{d}$ & gamma gliadin & BU-gamma5 & T.aestivum & 15 & 52 & 41 \\
\hline 62 & triticin & ACB41345 & T.aestivum & 15 & 49 & 26 \\
\hline 63 & isocitrate dehydrogenase & AMP82030 & T.aestivum & 11 & 41 & 27 \\
\hline 64 & serpin & EMS54555 & T. uratu & 34 & 102 & 68 \\
\hline 65 & serpin & CAB52709 & T.aestivum & 51 & 213 & 78 \\
\hline 66 & serpin & ACN59485 & T.aestivum & 35 & 109 & 66 \\
\hline 67 & serpin & ACN59485 & T.aestivum & 90 & 358 & 85 \\
\hline 68 & serpin & ACN59485 & T.aestivum & 55 & 162 & 78 \\
\hline 69 & serpin & ACN59484 & T.aestivum & 16 & 50 & 40 \\
\hline 70 & serpin & ACN59484 & T.aestivum & 25 & 75 & 48 \\
\hline 71 & serpin & AFC89429 & T.aestivum & 30 & 67 & 63 \\
\hline 72 & serpin & AFC89429 & T.aestivum & 87 & 268 & 86 \\
\hline 73 & serpin & AFC89429 & T.aestivum & 65 & 207 & 82 \\
\hline 74 & serpin & AFC89429 & T.aestivum & 11 & 36 & 32 \\
\hline 75 & glycosyltransferase 75 & ADK56176 & T.aestivum & 7 & 18 & 22 \\
\hline 76 & serpin & CAA90071 & T.aestivum & 31 & 123 & 61 \\
\hline 77 & serpin & CAA90071 & T.aestivum & 31 & 61 & 61 \\
\hline 78 & serpin & EMS46390 & T. uratu & 39 & 115 & 67 \\
\hline 79 & alpha gliadin & BAM08450 & T.aestivum & 10 & 23 & 34 \\
\hline $80^{\mathrm{b}, \mathrm{d}, \mathrm{f}}$ & alpha gliadin & CAY54134 & T.aestivum & 25 & 104 & 45 \\
\hline $81^{b}$ & ER molecular chaperone & AGN94840 & T.aestivum & 30 & 149 & 34 \\
\hline $82^{\mathrm{d}, \mathrm{f}}$ & gamma gliadin & BU-gamma-1 & T.aestivum & 29 & 164 & 50 \\
\hline $83^{\mathrm{d}, \mathrm{f}, \mathrm{g}}$ & alpha gliadin & BU-alpha-23 & T.aestivum & 38 & 107 & 63 \\
\hline $84^{d}$ & LMW-GS & ABC84361 & T.aestivum & 17 & 48 & 33 \\
\hline
\end{tabular}


Table 1 Predominant proteins identified by MS/MS in 2-DE protein spots from the KCl-soluble fraction of Butte 86 flour. All proteins had identification scores greater than 99\% probability with at least 4 peptides as determined by Scaffold. Positions of spots in 2-DE are shown in Fig. 2. Identities of all proteins found in each spot are reported in Additional file 1 (Continued)

\begin{tabular}{|c|c|c|c|c|c|c|}
\hline Spot Number & Predominant Protein & Accession $\#^{a}$ & Source & \# Unique peptides & \# Spectra & $\%$ Coverage \\
\hline 85 & no ID & & & & & \\
\hline $86^{f}$ & LMW-GS & ACJ76984 & T. dicoccoides & 7 & 25 & 16 \\
\hline $87^{\mathrm{d}, f}$ & gamma gliadin & AEA30015 & T.aestivum & 24 & 78 & 53 \\
\hline 88 & alpha gliadin & AAA96522 & T.aestivum & 11 & 24 & 42 \\
\hline 89 & peroxidase & XP_020147267 & Ae. tauschii & 38 & 173 & 53 \\
\hline 90 & peroxidase & AAM88383 & T.aestivum & 47 & 125 & 57 \\
\hline $91^{b, d, e}$ & peroxidase & AAM88383 & T.aestivum & 62 & 197 & 60 \\
\hline 92 & aldose 1 epimerase & XP_020180146 & Ae. tauschii & 9 & 24 & 22 \\
\hline 93 & globulin 3A & AFM30909 & T.aestivum & 19 & 57 & 25 \\
\hline 94 & $63 \mathrm{kd}$ globulin & XP-020163670 & Ae. tauschii & 24 & 88 & 27 \\
\hline 95 & farinin & XP_020147251 (BU-farinin-1) & Ae. tauschii & 9 & 49 & 31 \\
\hline 96 & 63 kd globulin & XP-020163670 & Ae. tauschii & 24 & 106 & 28 \\
\hline 97 & aspartic proteinase & XP_020157026 & Ae. tauschii & 9 & 27 & 19 \\
\hline $98^{9}$ & aspartic proteinase & XP_020157026 & Ae. tauschii & 5 & 13 & 13 \\
\hline 99 & cupincin & XP_020147625 & Ae. tauschii & 8 & 31 & 18 \\
\hline 100 & peroxidase & AAM88383 & T.aestivum & 13 & 36 & 34 \\
\hline $101^{b, d, h}$ & glyceraldehyde 3-P dehydrogenase & ALE18234 & T.aestivum & 49 & 164 & 65 \\
\hline $102^{b, d}$ & glyceraldehyde 3-P dehydrogenase & ANW11922 & T.aestivum & 79 & 230 & 92 \\
\hline $103^{b, d}$ & glyceraldehyde 3-P dehydrogenase & ALE18234 & T.aestivum & 37 & 85 & 68 \\
\hline $104^{\mathrm{b}, \mathrm{d}}$ & fructose bisphosphate aldolase & CDM85265 & T.aestivum & 60 & 197 & 73 \\
\hline $105^{\mathrm{b}, \mathrm{d}}$ & glyceraldehyde 3-P dehydrogenase & ABQ81648 & T.aestivum & 56 & 208 & 73 \\
\hline $106^{b, d, f}$ & alpha gliadin & CS-alpha-A1 & T.aestivum & 70 & 215 & 77 \\
\hline 107 & malate dehydrogenase & XP_020196362 & Ae. tauschii & 6 & 17 & 17 \\
\hline 108 & guanine nucleotide binding protein & CDM83867 & T.aestivum & 11 & 23 & 40 \\
\hline 109 & malate dehydrogenase & XP_020157252 & Ae. tauschii & 44 & 149 & 73 \\
\hline 110 & malate dehydrogenase & AAT64932 & T.aestivum & 21 & 53 & 54 \\
\hline 111 & desiccation-related protein & XP_020169835 & Ae. tauschii & 16 & 37 & 35 \\
\hline 112 & desiccation-related protein & XP_020169835 & Ae. tauschii & 11 & 34 & 31 \\
\hline 113 & lactoylglutathione lyase & XP_020185375 & Ae. tauschii & 13 & 38 & 41 \\
\hline 114 & lactoylglutathione lyase & XP_020185375 & Ae. tauschii & 51 & 149 & 79 \\
\hline 115 & lactoylglutathione lyase & XP_020185375 & Ae. tauschii & 16 & 54 & 44 \\
\hline 116 & lactoylglutathione lyase & EMS56223 & T. uratu & 7 & 19 & 21 \\
\hline 117 & glucose and ribitol dehydrogenase & XP_020188730 & Ae. tauschii & 16 & 41 & 46 \\
\hline 118 & glucose and ribitol dehydrogenase & XP_020188730 & Ae. tauschii & 28 & 107 & 61 \\
\hline 119 & glucose and ribitol dehydrogenase & XP_020188730 & Ae. tauschii & 31 & 127 & 67 \\
\hline 120 & glucose and ribitol dehydrogenase & XP_020188730 & Ae. tauschii & 29 & 101 & 65 \\
\hline 121 & proteosome subunit alpha type 1 & BAJ85935 & Hordeum vulgare & 10 & 28 & 39 \\
\hline 122 & glucose and ribitol dehydrogenase & BAJ85825 & Hordeum vulgare & 15 & 64 & 39 \\
\hline 123 & dehydrin & AOM63239 & T.aestivum & 12 & 29 & 61 \\
\hline 124 & purinin & ADA62372 (BU-purinin-3) & T.aestivum & 14 & 105 & 40 \\
\hline 125 & 19 kd globulin & XP_020162707 & Ae. tauschii & 12 & 65 & 61 \\
\hline 126 & oil body associated protein & XP_020196637 & Ae. tauschii & 24 & 74 & 81 \\
\hline
\end{tabular}


Table 1 Predominant proteins identified by MS/MS in 2-DE protein spots from the KCl-soluble fraction of Butte 86 flour. All proteins had identification scores greater than 99\% probability with at least 4 peptides as determined by Scaffold. Positions of spots in 2-DE are shown in Fig. 2. Identities of all proteins found in each spot are reported in Additional file 1 (Continued)

\begin{tabular}{|c|c|c|c|c|c|c|}
\hline Spot Number & Predominant Protein & Accession $\#^{\mathrm{a}}$ & Source & \# Unique peptides & \# Spectra & $\%$ Coverage \\
\hline 127 & 19 kd globulin & XP_020162707 & Ae. tauschii & 9 & 62 & 50 \\
\hline 128 & xylanase inhibitor & AGN71004 & T.aestivum & 34 & 127 & 64 \\
\hline 129 & globulin-1 & ABG68034 & T.aestivum & 12 & 55 & 46 \\
\hline 130 & chitinase & XP_020181917 & Ae. tauschii & 19 & 108 & 41 \\
\hline 131 & globulin & AAR95703 & T. turgidum & 11 & 65 & 50 \\
\hline 132 & globulin & AAR95703 & T. turgidum & 10 & 72 & 50 \\
\hline 133 & tritin & BAA02948 & T.aestivum & 53 & 316 & 75 \\
\hline 134 & farinin & XP_020147251 (BU-farinin-1) & Ae. tauschii & 7 & 35 & 21 \\
\hline 135 & secretory protein & XP_020161391 & Ae. tauschii & 9 & 23 & 38 \\
\hline 136 & secretory protein & XP_020161391 & Ae. tauschii & 5 & 14 & 29 \\
\hline 137 & cyclophilin A & AAK49426 & T.aestivum & 14 & 84 & 57 \\
\hline 138 & chitinase & XP_020181917 & Ae. tauschii & 15 & 28 & 32 \\
\hline 139 & chitinase & AAX83263 & T.aestivum & 20 & 73 & 48 \\
\hline 140 & AAl & prf 1207200A (WASI BU-1) & T.aestivum & 39 & 234 & 82 \\
\hline 141 & AAl & prf 1207200A (WASI BU-1) & T.aestivum & 14 & 72 & 51 \\
\hline 142 & AAl & prf 1207200A (WASI BU-1) & T.aestivum & 31 & 112 & 71 \\
\hline 143 & peroxiredoxin & ACE82290 & T.aestivum & 21 & 91 & 67 \\
\hline 144 & 1-cys peroxiredoxin & AAQ74769 & T.aestivum & 21 & 73 & 65 \\
\hline 145 & purinin & EMS62569 (BU-purinin-1) & T. urartu & 22 & 98 & 68 \\
\hline 146 & purinin & EMS62569 (BU-purinin-1) & T. urartu & 9 & 37 & 52 \\
\hline 147 & triosephosphate isomerase & CDM81250 & T.aestivum & 31 & 102 & 70 \\
\hline 148 & oil body associated protein & XP_020147075 & Ae. tauschii & 30 & 103 & 84 \\
\hline 149 & late embryogenesis abundant protein & XP_020193122 & Ae. tauschii & 10 & 22 & 55 \\
\hline 150 & late embryogenesis abundant protein & XP_020193122 & Ae. tauschii & 14 & 39 & 62 \\
\hline 151 & late embryogenesis abundant protein & BAK04960 & Hordeum vulgare & 6 & 16 & 28 \\
\hline 152 & glutathionine-S-transferase & XP_020196644 & Ae. tauschii & 15 & 57 & 49 \\
\hline 153 & glutathionine-S-transferase & XP_020196644 & Ae. tauschii & 51 & 214 & 60 \\
\hline 154 & glutathionine-S-transferase & XP_020196644 & Ae. tauschii & 21 & 65 & 53 \\
\hline 155 & Iysosomal thiol reductase & XP_020155412 & Ae. tauschii & 15 & 73 & 52 \\
\hline 156 & nucleoside diphosphate kinase & EMS63724 & T. urartu & 5 & 16 & 41 \\
\hline 157 & AAl & AAR10959 (WTAI-CM3 BU-1) & T.aestivum & 37 & 388 & 74 \\
\hline 158 & no ID & & & & & \\
\hline 159 & AAl & CAl84642 (WDAI BU-1) & T.aestivum & 8 & 24 & 66 \\
\hline 160 & no ID & & & & & \\
\hline 161 & no ID & & & & & \\
\hline 162 & no ID & & & & & \\
\hline 163 & ubiquitin & AAP50253 & T.aestivum & 10 & 56 & 83 \\
\hline 164 & AAl & AAV39514 (WDAI BU-1) & T.aestivum & 26 & 244 & 100 \\
\hline 165 & AAl & AAV39517 (WDAI BU-1) & T.aestivum & 22 & 101 & 88 \\
\hline 166 & AAl & sp P16851 (WTAl-CM2 BU-1) & T.aestivum & 24 & 107 & 74 \\
\hline 167 & superoxide dismutase & AFF27607 & T.aestivum & 18 & 61 & 66 \\
\hline 168 & globulin-1 & EMS62417 & T. urartu & 12 & 53 & 15 \\
\hline
\end{tabular}


Table 1 Predominant proteins identified by MS/MS in 2-DE protein spots from the $\mathrm{KCl}$-soluble fraction of Butte 86 flour. All proteins had identification scores greater than 99\% probability with at least 4 peptides as determined by Scaffold. Positions of spots in 2-DE are shown in Fig. 2. Identities of all proteins found in each spot are reported in Additional file 1 (Continued)

\begin{tabular}{|c|c|c|c|c|c|c|}
\hline Spot Number & Predominant Protein & Accession $\#^{a}$ & Source & \# Unique peptides & \# Spectra & $\%$ Coverage \\
\hline 169 & $\mathrm{AAl}$ & ABO45967 (WMAI BU-1) & T.aestivum & 25 & 135 & 99 \\
\hline 170 & AAl & ABI54484 (WDAI BU-3) & T. dicoccoides & 14 & 58 & 96 \\
\hline 171 & $\mathrm{AAl}$ & ABO45967 (WMAI BU-1) & T.aestivum & 19 & 116 & 98 \\
\hline 172 & AAl & AAV91972 (WDAI BU-4) & T.aestivum & 37 & 198 & 100 \\
\hline 173 & $\mathrm{AAl}$ & ACM41418 (WTAl-CM16 BU-1) & T. macha & 34 & 228 & 80 \\
\hline 174 & AAl & CAA42453 (WTAl-CM-17 BU-1) & T.aestivum & 25 & 133 & 77 \\
\hline 175 & AAl & CAA42453 (WTAI-CM-17 BU-1) & T.aestivum & 13 & 59 & 50 \\
\hline 176 & $\mathrm{AAl}$ & ABO45967 (WMAI BU-1) & T.aestivum & 4 & 17 & 36 \\
\hline 177 & AAl & sp P16851 (WTAl-CM2 BU-1) & T.aestivum & 7 & 25 & 44 \\
\hline 178 & homocysteine methyltransferase & EMS51950 & T. urartu & 20 & 76 & 27 \\
\hline $189^{\mathrm{d}, \mathrm{f}}$ & alpha gliadin & BU-alpha-3 & T.aestivum & 15 & 44 & 35 \\
\hline $190^{\mathrm{d}, \mathrm{e}}$ & alpha gliadin & BAM08458 & T.aestivum & 23 & 73 & 50 \\
\hline $191^{b, d}$ & alpha gliadin & BU-alpha-4 & T.aestivum & 19 & 53 & 46 \\
\hline
\end{tabular}

${ }^{a}$ Accession numbers beginning with BU are from Butte 86 and CS are from Chinese Spring. All other accession numbers are from NCBI. The Butte 86 accession is shown in parentheses in cases where a sequence from NCBI is identical to a sequence from Butte 86 . Gliadin, purinin and farinin sequences from Butte 86 are from [19]. AAl sequences from Butte 86 are from [22]

$\mathrm{b}$ a gluten protein with less than half the number of unique peptides found in the predominant protein was also identified in spot

$c$ also contains an omega gliadin

d replicate sample was digested with thermolysin and analyzed by MS/MS

e also contains a LMW-GS

${ }^{f}$ also contains a non-gluten protein

${ }^{\mathrm{g}}$ also contains a gamma gliadin

h also contains an alpha gliadin

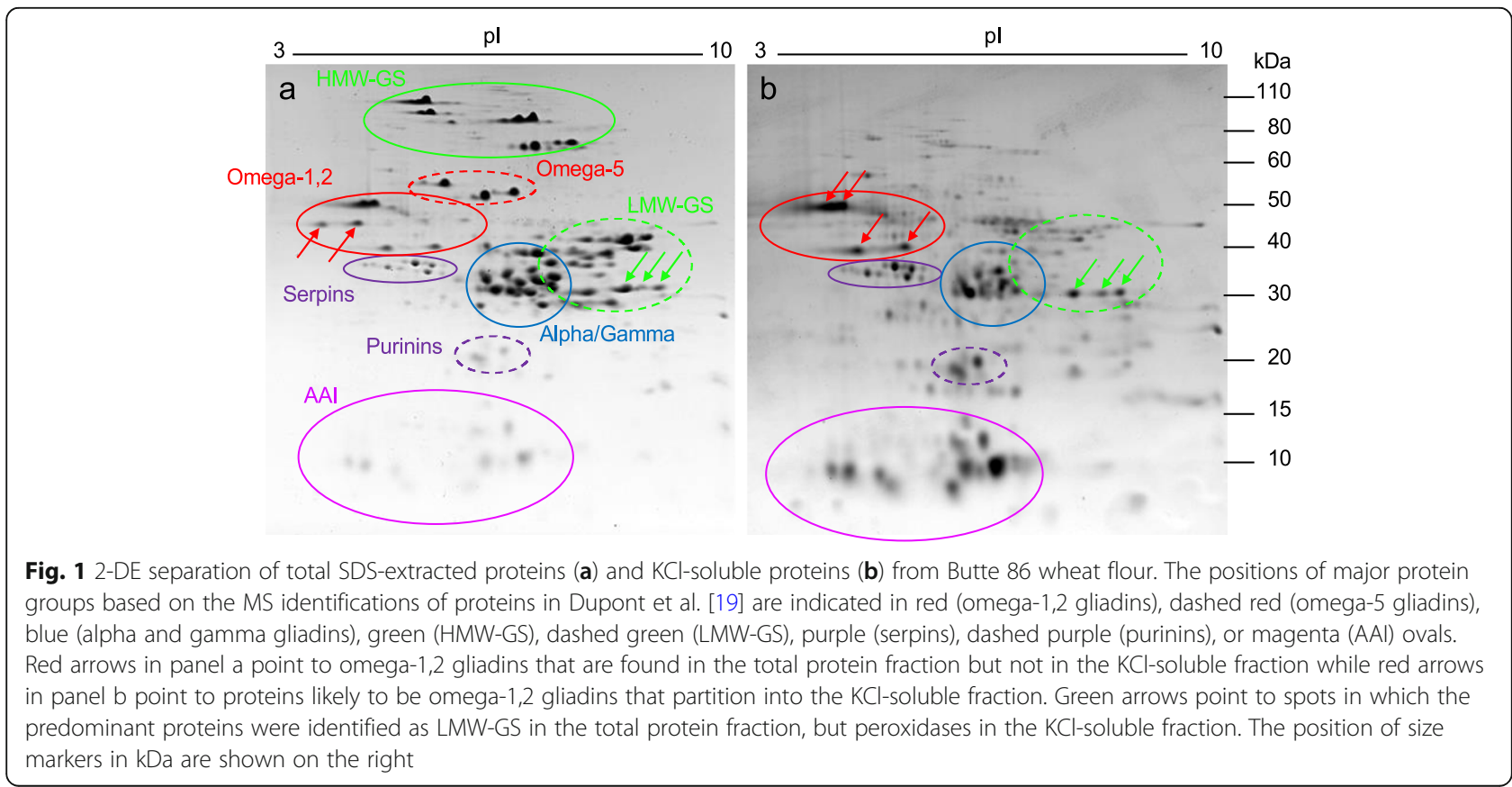




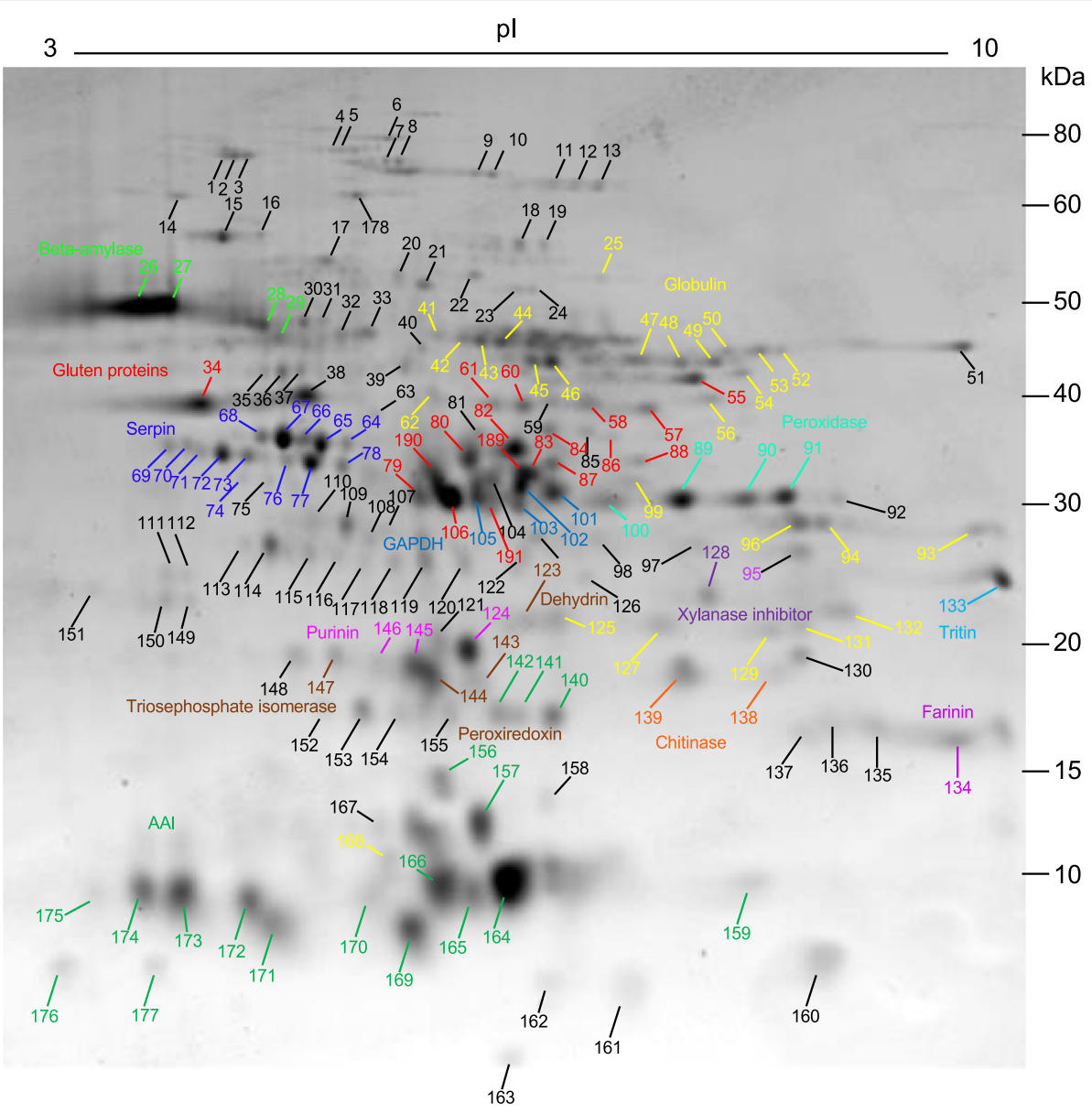

Fig. 2 Identification of proteins in a $\mathrm{KCl}$-soluble fraction of wheat flour. Spots in which the predominant proteins were gluten proteins are labeled in red. Spots in which the predominant proteins were non-gluten proteins that are either known allergens or likely to be immunogenic are labeled in bright green (beta-amylase), dark blue (serpins), medium blue (glyceraldehyde-3-phosphate dehydrogenase (GAPDH), yellow (globulins), teal (peroxidase), magenta (purinins), orange (chitinase), purple (xylanase inhibitor), light blue (tritin), light purple (farinin), brown (dehydrin, peroxiredoxin, triosephosphate isomerase), or green (AAl). Protein identifications can be found in Table 1 and Additional file 1

proteins in the $\mathrm{KCl}$-soluble fraction (Fig. $1 \mathrm{~b})$ were located in the AAI, omega-1,2 gliadin and alpha and gamma gliadin regions of the gel. Additionally, proteins in the serpin and purinin regions were notably more abundant in the $\mathrm{KCl}$-soluble fraction than in the total protein fraction.

One-hundred-eighty-one spots were excised from 2-D gels of the $\mathrm{KCl}$-soluble proteins and analyzed by MS/MS (Fig. 2). One hundred seventy-three of the spots yielded valid identifications. MS/MS coverage of the proteins ranged from 8 to $100 \%$ with an average of $49 \%$. Sixty-one different protein types and 111 sequences were identified as the predominant proteins in these spots (Table 1). The predominant proteins in 26 spots were globulins (Fig. 2). Nine different globulin sequences were identified. Seventeen spots were identified as AAI and 11 different sequences were found. Fourteen spots were serpins and seven different sequences were identified. In addition, the predominant proteins in multiple spots were identified as glucose and ribitol dehydrogenase (6), embryonic protein DC-8 (5), beta-amylase (4), enolase (4), GAPDH (4), lactoylglutathione lyase (4), peroxidase (4), chitinase (3), glutathioneS-transferase (3), late embryogenesis abundant protein (3), malate dehydrogenase (3), pyruvate phosphate dikinase (3), and purinin (3). Forty other non-gluten protein types were identified as the predominant proteins in either one or two 2-DE spots (Table 1). Only eight of these were identified previously in the total protein fraction [19].

In some cases, gluten proteins were separated effectively from non-gluten proteins on the basis of solubility. For example, peroxidase was the predominant protein in three moderately abundant spots $(89,90,91)$ in the LMW-GS region of the gel from the $\mathrm{KCl}$-soluble fraction (green arrows in Figs. 1b and 2). In the total protein fraction of Dupont et al. [19], the predominant proteins in these three spots were LMW-GS (indicated with green arrows in Fig. 1a). The absence of most LMW-GS in the KCl-soluble fraction also made it easier to visualize many of the minor globulins that are found in this region of the gel (labeled in yellow in Fig. 2). 
Despite the fact that most gluten proteins are not readily soluble in salt solutions, gluten proteins were the predominant proteins identified in 18 spots in the $\mathrm{KCl}$ fraction (Fig. 2). Eight spots were alpha gliadins, four were gamma gliadins, one was an omega gliadin and five were LMWGS (Table 1). Fourteen of these spots also contained nongluten proteins $(34,55,60,61,80,82,83,84,86,87,106$, $189,190,191)$. Gluten proteins also were detected as minor components of 14 spots (Table 1, Additional file 1).

Four major spots $(26,27,34,38)$ in the omega-1,2 gliadin region of the gel were confounding (indicated with red arrows in Fig. 1b, Fig. 2). The predominant proteins identified in spots 26 and 27 were beta-amylase (Table 1). However, protein disulfide isomerase and omega-1,2 gliadin BAN29067 also were identified in these spots (Additional file 1). Spot 34 was identified as omega-1,2 gliadin AKB95614 whereas spot 38 contained both enolase and omega-1,2 gliadin AKB95614. Interestingly, two other spots previously identified as omega-1,2 gliadins (shown with red arrows in Fig. 1a) did not appear in the $\mathrm{KCl}$-soluble fraction even though their sequences were very similar to the omega-1,2 gliadins found in spots 26 and 27 [16].

A number of spots in the region of the gel containing alpha and gamma gliadins also were problematic. Three minor spots in this region contained only alpha gliadins $(79,88,191)$ while two minor spots contained only gamma gliadins $(60,61)$. However, five moderately abundant spots contained both an alpha gliadin as the predominant protein and GAPDH $(83,106,189,190,191)$. Additionally, the predominant proteins in four other spots were GADPH, but MS/MS evidence also pointed to the presence of alpha gliadins in these spots (101, 102, 103, 105) (Fig. 2, Additional file 1).

To estimate the amounts of different gluten proteins that were carried over into the $\mathrm{KCl}$-soluble protein fraction, we examined the profiles of flour proteins from transgenic lines in which the expression of either the omega-1,2 gliadins or the alpha gliadins was suppressed by RNA interference $[16,17]$. Detailed proteomic analyses of total protein fractions from flour of the transgenic lines revealed that all omega-1,2 gliadins were absent in line SA-30-118a-5 [16] while all alpha gliadins were absent in line SA-35a-124j [17]. In 2-D gels of the $\mathrm{KCl}$-soluble protein fraction, spots $26,27,34,38$ containing omega-1,2 gliadins were major spots in the nontransgenic flour (Fig. 3a). Spot 26 was significantly reduced while other spots were not apparent in transgenic line SA-30-118a-5 (Fig. 3b), suggesting that significant amounts of certain omega-1,2 gliadins partition into the $\mathrm{KCl}$-soluble fractions and that beta-amylase and protein disulfide isomerase are low abundance proteins. Within the alpha gliadin region of the gel (Fig. 3a, c), several spots in which either alpha gliadins (79) or alpha gliadins and GAPDH $(83,106,189)$ were identified in the $\mathrm{KCl}$ fraction from Butte 86 were not apparent in transgenic line SA-35a-124j, suggesting that alpha gliadins comprise the bulk of the protein in these spots. Several other spots containing both alpha gliadin and GAPDH were present in similar amounts in Butte 86 and the transgenic line $(101,102,103,105,190,191)$, suggesting that most of the protein in these spots is GAPDH and that this protein is fairly abundant in the $\mathrm{KCl}$ fraction. Interestingly, GADPH was not identified in the total protein fraction in the Dupont et al. (2011) study [19].

\section{Discussion}

A number of non-gluten proteins that are abundant in the $\mathrm{KCl}$-soluble protein fraction are well-separated from the major gluten proteins and can be identified unambiguously by MS/MS in a total SDS protein extract of Butte 86 flour [19]. These include the serpins, purinins and AAI. However, other non-gluten proteins are overshadowed by the more abundant gluten proteins. In 2-DE gels of total protein extracts, betaamylases are hidden by omega-1,2 gliadins, GAPDHs are masked by alpha gliadins, and peroxidases and some globulins are obscured by LMW-GS. In this study, non-gluten proteins were preferentially extracted from wheat flour using $\mathrm{KCl}$ and 57 different types of non-gluten proteins were identified, including 14 types that are known or suspected immunogenic proteins. Although most gluten proteins are insoluble in $\mathrm{KCl}$, we observed some carry-over of the abundant gluten proteins in the $\mathrm{KCl}$ fraction. This was not unexpected and, in contrast to other studies [8-11], was likely noted because protein spots were digested separately with trypsin and chymotrypsin before analysis by MS/MS, thereby facilitating the identification of both non-gluten proteins that are readily digested with trypsin and gluten proteins that have few tryptic peptide cleavage sites. For example, 276 tryptic peptides were identified for beta-amylase in spot 27 and 62 tryptic peptides were identified for enolase in spot 38. The omega-1,2 gliadins in these two spots did not yield any tryptic peptides, but were identified on the basis of 81 and 41 chymotryptic peptides, respectively. Likewise, 72 tryptic peptides were identified for GAPDH in spot 101. Although the alpha gliadin in the same spot did not yield any tryptic peptides, it was identified by 24 chymotryptic peptides (Additional file 2). In general, protein spots that contained either gamma gliadins or LMW-GS were relatively minor while spots containing certain omega-1,2 gliadins or alpha gliadins were quite prominent in the $\mathrm{KCl}$-soluble fraction. Interestingly, two spots found in the omega-1,2 gliadin region of the total protein fraction but not in the $\mathrm{KCl}$-soluble fraction were 

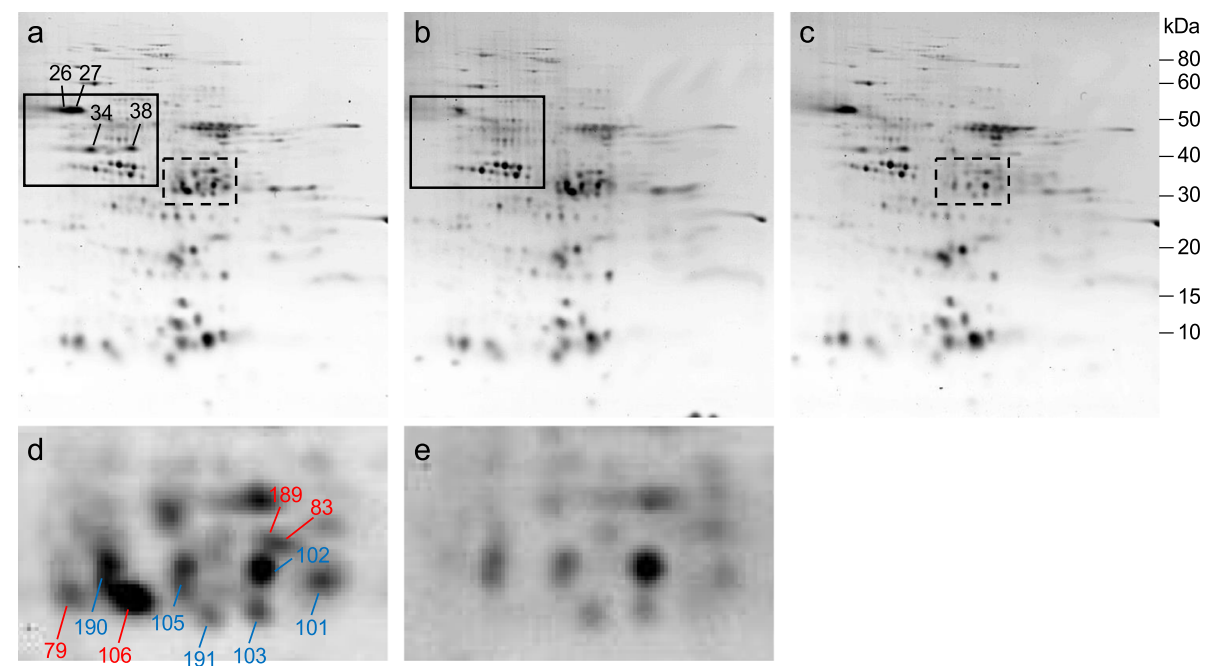

Fig. $3 \mathrm{KCl}$-soluble flour proteins in Butte 86 (a) and transgenic lines SA-30-118a-5 (b) and SA-35a-124j (c). The solid box highlights regions of the gels containing the omega-1,2 gliadins while the dashed box highlights regions of gels containing the alpha gliadins. The alpha gliadin regions from Butte 86 and SA-35a-124j were enlarged in panels (d) and (e), respectively. Spots labeled in red in panel d were significantly reduced or absent in SA-35a-124j and are predominantly alpha gliadins that partition into the KCl fraction. Spots labeled in blue in panel (d) were also found in the $\mathrm{KCl}$ fraction of SA-35a-124j and contain mostly GAPDH

identified previously as omega-1,2 gliadins that contain single cysteine residues [16]. These cysteine residues likely enable the proteins to be incorporated into glutenin polymers, rendering them insoluble in aqueous solutions even though their sequences are otherwise quite similar to omega-1,2 gliadins that partition into the $\mathrm{KCl}$ fraction. Indeed, in past experiments, these omega-1,2 gliadins were accumulated preferentially in the polymer fractions of Butte 86 wheat flour [24].

The availability of transgenic lines missing either the omega-1,2 gliadins or the alpha gliadins made it possible to estimate the abundance of specific non-gluten proteins that overlap with gluten proteins in the $\mathrm{KCl}$ soluble fraction. These analyses demonstrated that betaamylase and protein disulfide isomerase are low abundance proteins while GAPDH is a protein of moderate abundance.

Di Francesco et al. [25] recently used a shotgun approach to characterize the $\mathrm{KCl}$-soluble proteins from old and modern durum wheats. In their study, proteins were prepared from mature kernels rather than milled flour and the protein extracts were digested only with trypsin. Analysis of the resulting spectral data used a database of protein sequences from Triticum, Oryza, Hordeum, Avena, Secale, Maize and Brachypodium downloaded from the UniProt database. Interestingly, only $21 \%$ of identified proteins were from Triticum, $10.8 \%$ from Hordeum and 1\% from Secale. In the current study, proteins were digested individually with either trypsin or chymotrypsin and spectra were searched against a database containing all Triticeae sequences from NCBI along with a selection of sequences from Chinese Spring and Butte 86 . Not surprisingly, many of the same types of proteins, including likely allergens, were identified in both studies. However, it is curious that Di Francesco et al. identified only one globulin in their study while nine different globulin sequences were identified in 262 DE spots in the current study. Likewise, Di Francesco et al. identified only three different serpins, while seven different sequences in 14 spots were found in the current study. They did, however, report a number of alpha and gamma gliadins as well as HMW-GS and LMW-GS in the $\mathrm{KCl}$ fraction, confirming the carryover of gluten proteins into this fraction that was also observed in our analysis. It is likely that they would have identified more gluten proteins in this fraction if proteins had been digested with both trypsin and chymotrypsin prior to MS analysis.

\section{Conclusions}

The proteomic map of $\mathrm{KCl}$-soluble flour proteins generated in this study complements the map of total flour proteins from Butte 86 described by Dupont et al. [19] and serves as a reference map that, combined with 2-D immunoblots using sera from wellcharacterized patients, should provide new insight into non-gluten proteins that are responsible for immunogenic responses to wheat flour and lead to a 
better understanding of the complement of proteins associated with different types of allergies. Transgenic lines missing specific groups of gluten proteins should be of value in confirming whether reactivity with patient antibodies is due to non-gluten proteins or to gluten proteins that are carried over into the $\mathrm{KCl}$ soluble fraction. This information is critical for future efforts to develop wheat with reduced immunogenic potential.

\section{Supplementary information}

Supplementary information accompanies this paper at https://doi.org/10. 1186/s12953-020-00164-6.

Additional file 1. Proteins identified by MS/MS in 2-DE protein spots from the $\mathrm{KCl}$-soluble fraction of Butte 86 flour. The positions of the spots are shown in Figure 2. For each spot, the predominant protein and all proteins that contained at least half the number of unique peptides as the predominant protein are highlighted in yellow. Proteins that contained less than half the number of unique peptides found in the predominant protein are highlighted in blue. Gluten proteins that contained less than half the number of unique peptides found in the predominant protein are highlighted in green.

Additional file 2. MS/MS data for individual protein spots shown in Figure 2. Scaffold data for all proteins for which the number of unique peptides was equal to or greater than half the number assigned to the predominant protein are shown.

\section{Abbreviations}

2-DE: Two-dimensional gel electrophoresis; AAl: Alpha amylase/trypsin inhibitors; CD: Celiac disease; GAPDH: Glyceraldehyde 3-phosphate dehydrogenase; HMW-GS: High molecular weight glutenin subunit; LMW-GS: LoW molecular glutenin subunit; MS/MS: Tandem mass spectrometry; NCWS: Nonceliac wheat sensitivity

\section{Acknowledgements}

The authors thank Leslie Harden and Melissa Erickson-Beltran for expert advice and assistance with mass spectrometry work.

\section{Authors' contributions}

SA designed experiments, analyzed the data and wrote the manuscript, $\mathrm{H}$ CC was responsible for 2-DE analyses and AS-B did the MS/MS work. All authors read and approved the manuscript.

\section{Funding}

Research was funded by USDA Agricultural Research Service CRIS Project 2030-21430-014-00D. USDA is an equal opportunity provider and employer. Mention of a specific product name by the United States Department of Agriculture does not constitute an endorsement and does not imply a recommendation over other suitable products.

\section{Availability of data and materials}

The dataset generated in this study has been deposited in the ProteomeXchange Consortium (http://proteomecentral.proteomexchange. org) via the PRIDE partner repository.

\section{Ethics approval and consent to participate}

Not applicable.

\section{Consent for publication}

Not applicable.

\section{Competing interests}

The authors declare that they have no competing interests.

\section{Author details}

${ }^{1}$ Western Regional Research Center, United States Department of Agriculture-Agricultural Research Service, Albany, CA, USA. ${ }^{2}$ Hamburg School of Food Science, Institute of Food Chemistry, University of Hamburg, Hamburg, Germany.

Received: 23 January 2020 Accepted: 21 July 2020

Published online: 01 August 2020

\section{References}

1. Scherf KA. Immunoreactive cereal proteins in wheat allergy, non-celiac gluten/ wheat sensitivity (NCGS) and celiac disease. Curr Opin Food Sci. 2019;25:35-41..

2. Scherf KA, Koehler P, Wieser H. Gluten and wheat sensitivities - an overview. J Cereal Sci. 2016;67:2-11..

3. Sollid LM, Qiao S-W, Anderson RP, Gianfrani C, Konig F. Nomenclature and listing of celiac disease relevant gluten T-cell epitopes restricted by HLA-DQ molecules. Immunogenetics. 2012;64:455-60..

4. Tye-Din JA, Stewart JA, Dromey JA, Beissbarth T, van Heel DA, Tatham A, et al. Comprehensive, quantitative mapping of T cell epitopes in gluten in celiac disease. Sci Transl Med. 2010:4:41ra51.

5. Battais F, Courcoux P, Popineau Y, Kanny G, Moneret-Vautrin DA, DeneryPapini S. Food allergy to wheat: differences in immunoglobulin E-binding proteins as a function of age or symptoms. J Cereal Sci. 2005;42:109-17.

6. Palosuo K, Alenius H, Varjonen E, Koivuluhta M, Mikkola J, Keskinen H, et al. A novel wheat gliadin as a cause of exercise-induced anaphylaxis. J Allergy Clin Immunol. 1999:103:912-7..

7. Battais F, Pineau F, Popineau Y, Aparicio C, Kanny G, Guerin L, Moneret-Vautrin DA, Denery-Papini S. Food allergy to wheat: identification of immunogloglin $E$ and immunoglobulin G-binding proteins with sequential extracts and purified proteins from wheat flour. Clin Exp Allergy. 2003:33:962-70..

8. Akagawa M, Handoyo T, Ishii T, Kumazawa S, Morita N, Suyama K. Proteomic analysis of wheat flour allergens. J Agric Food Chem. 2007;55:6863-70..

9. Pastorello EA, Farioli L, Conti A, Pravettoni V, Bonomi S, lametti S, et al. Wheat IgE-mediated food allergy in European patients: a-amylase inhibitors, lipid transfer proteins and low-molecular-weight glutenins. Int Arch Allergy Immunol. 2007;144:10-22..

10. Sotkovsky P, Hubalek M, Hernychova L, Novak P, Havranova M, Setinova I, et al. Proteomic analysis of wheat proteins recognized by lgE antibodies of allergic patients. Proteomics. 2008;9:1677-91..

11. Sotkovsky P, Sklenar J, Halada P, Cinova J, Setinova I, Kainarova A, et al. A new approach to the isolation and characterization of wheat flour. Clin Exp Allergy. 2011:41:1031-43..

12. Huebener S, Tanaka CK, Uhde M, Zone JJ, Vensel WH, Kasarda DD, et al. Specific nongluten proteins of wheat are novel target antigens in celiac disease humoral response. J Proteome Res. 2015;14:503-11..

13. Junker Y, Zeissig S, Kim S-J, Barisani D, Wieser H, Leffler DA, et al. Wheat amylase trypsin inhibitors drive intestinal inflammation via activation of tolllike receptor 4. J Exp Med. 2012;209:2395-408..

14. MacFarlane AJ, Burghardt KM, Kelly J, Simell T, Simell O, Altosaar I, et al. A type 1 diabetes-related protein from wheat (Triticum aestivum). J Biol Chem. 2003;278:54-63.

15. Altenbach SB, Tanaka CK, Pineau F, Lupi R, Drouet M, Beaudouin E, Morisset M, Denery-Papini S. Assessment of the allergenic potential of transgenic wheat (Triticum aestivum) with reduced levels of $\omega-5$ gliadins, the major sensitizing allergen in wheat-dependent exercise-induced anaphylaxis. J Agric Food Chem. 2015;63:9323-32..

16. Altenbach SB, Chang H-C, Yu XB, Seabourn BW, Green PH, Alaedini A. Elimination of omega-1,2 gliadins from bread wheat (Triticum aestivum) flour: effects on immunogenic potential and end-use quality. Front Plant Sci. 2019;10:580..

17. Altenbach $\mathrm{SB}$, Chang $\mathrm{H}-\mathrm{C}$, Rowe $\mathrm{MH}$, Yu XB, Simon-Buss A, Seabourn BW, Green PH, Alaedini A. Reducing the immunogenic potential of wheat flour: silencing of alpha gliadin genes in a U.S. wheat cultivar. Front Plant Sci. 2020;11:20..

18. Hurkman WJ, Tanaka CK. Improved methods for separation of wheat endosperm proteins and analysis by two-dimensional gel electrophoresis. J Cereal Sci. 2004:40:295-9..

19. Dupont FM, Vensel WH, Tanaka CK, Hurkman WJ, Altenbach SB. Deciphering the complexities of the wheat flour proteome using quantitative twodimensional electrophoresis, three proteases and tandem mass spectrometry. Proteome Sci. 2011;9:10.

20. Huo N, Zhang S, Zhu T, Dong L, Mohr T, Hu T, et al. Gene duplication and evolution dynamics in the homeologous regions harboring multiple prolamin and resistance gene families in hexaploid wheat. Front Plant Sci. 2018a;9:673.. 
21. Huo N, Zhu T, Altenbach S, Dong L, Wang Y, Mohr T, et al. Dynamic evolution of a-gliadin prolamin gene family in homeologous genomes of hexaploid wheat. Sci Rep. 2018b;8:5181.

22. Altenbach SB, Vensel WH, Dupont FM. The spectrum of low molecular weight alpha-amylase/protease inhibitor genes expressed in the US bread wheat cultivar Butte 86. BMC Research Notes. 2011:4:242.

23. Perez-Riverol Y, Csordas A, Bai J, Bernal-Llinares M, Hewapathirana S, Kundu, et al. The PRIDE database and related tools and resources in 2019: improving support for quantification data. Nucleic Acids Res. 2019;47(D1):D442-50.

24. Vensel WH, Tanaka CK, Altenbach SB. Protein composition of wheat gluten polymer fractions determined by quantitative two-dimensional gel electrophoresis and tandem mass spectrometry. Proteome Sci. 2014;12:8..

25. Di Francesco A, Saletti R, Cunsolo V, Svensson B, Muccilli V, De Vita P, Foti S. Qualitative proteomic comparison of metabolic and CM-like protein fractions in old and modern wheat Italian genotypes by a shotgun approach. J Proteome. 2020;211:103530..

\section{Publisher's Note}

Springer Nature remains neutral with regard to jurisdictional claims in published maps and institutional affiliations.

Ready to submit your research? Choose BMC and benefit from:

- fast, convenient online submission

- thorough peer review by experienced researchers in your field

- rapid publication on acceptance

- support for research data, including large and complex data types

- gold Open Access which fosters wider collaboration and increased citations

- maximum visibility for your research: over $100 \mathrm{M}$ website views per year

At BMC, research is always in progress.

Learn more biomedcentral.com/submissions 